Revue de droit comparé du travail et de la sécurité sociale

2| 2018

Harcèlement moral au travail en jurisprudence

comparée

\title{
Le statut juridique du harcèlement psychologique aux États-Unis
}

\section{Camille Hébert}

\section{CpenEdition}

Journals

Édition électronique

URL : https://journals.openedition.org/rdctss/1952

DOI : $10.4000 /$ rdctss. 1952

ISSN : 2262-9815

Éditeur

Centre de droit comparé du travail et de la sécurité sociale

Édition imprimée

Date de publication : 1 juin 2018

Pagination : 86-93

ISSN : 2117-4350

Référence électronique

L. Camille Hébert, «Le statut juridique du harcèlement psychologique aux États-Unis », Revue de droit comparé du travail et de la sécurité sociale [En ligne], 2 | 2018, mis en ligne le 01 novembre 2021, consulté le 12 novembre 2021. URL : http://journals.openedition.org/rdctss/1952 ; DOI : https:// doi.org/10.4000/rdctss. 1952

\section{(c) (i) $\ominus$}

Revue de droit comparé du travail et de la sécurité sociale est mise à disposition selon les termes de la Licence Creative Commons Attribution - Pas d'Utilisation Commerciale - Pas de Modification 4.0 International. 


\section{LE STATUT JURIDIQUE DU HARCÈLEMENT PSYCHOLOGIQUE AUX ÉTATS-UNIS}
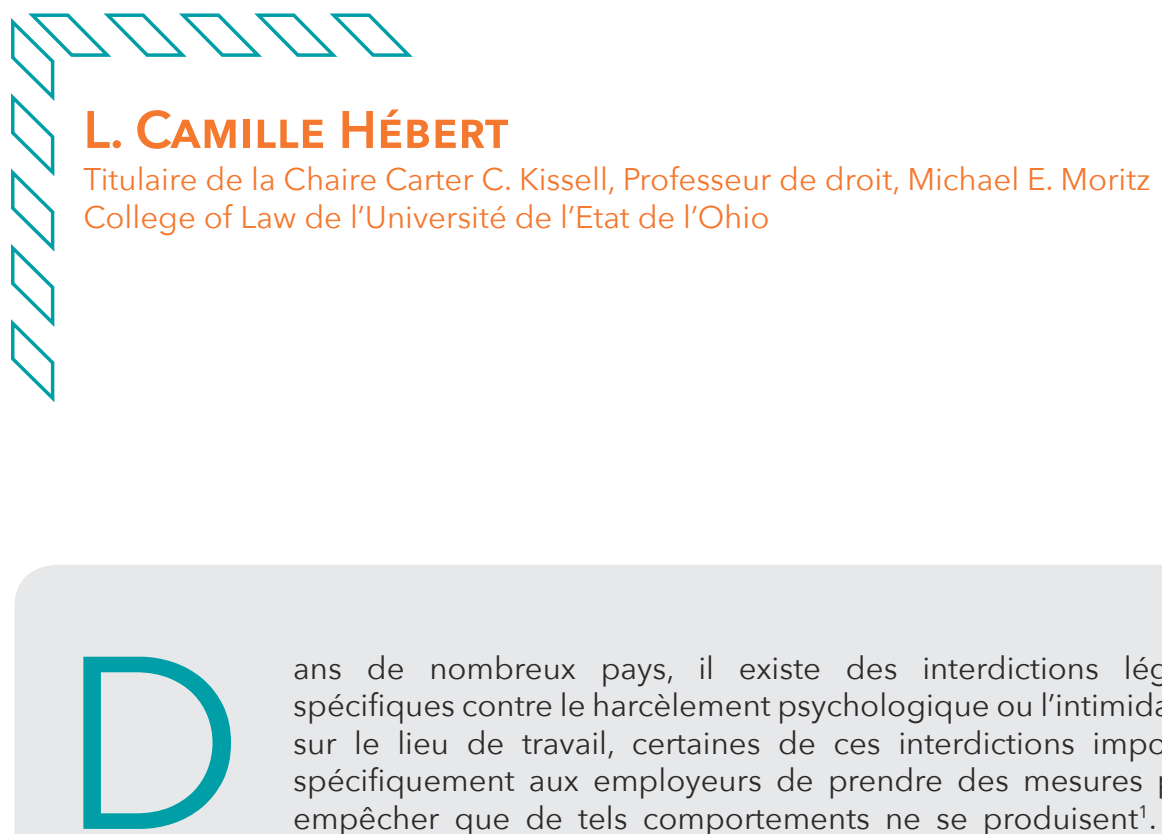

ans de nombreux pays, il existe des interdictions légales spécifiques contre le harcèlement psychologique ou l'intimidation sur le lieu de travail, certaines de ces interdictions imposant spécifiquement aux employeurs de prendre des mesures pour empêcher que de tels comportements ne se produisent ${ }^{1}$. Aux Etats-Unis, la loi est à la fois moins spécifique et plus aléatoire en ce qui concerne l'existence d'un devoir imposé aux employeurs face à ce type de comportement. La raison de cette différence réside dans le fait que la loi aux Etats-Unis n'interdit pas spécifiquement le harcèlement psychologique au travail. Les employeurs sont simplement tenus d'agir ; ils ne peuvent être tenus responsables que dans les cas où ils omettent d'agir et dans la mesure où le harcèlement psychologique viole une autre interdiction légale. Nonobstant des efforts déployés pour adopter une loi visant spécifiquement à interdire le harcèlement psychologique en milieu de travail, il n'existe toujours pas de législation spécifique à ce jour².

1 En France, par exemple, le Code pénal et le Code du travail interdisent le harcèlement moral et les employeurs ont un devoir spécifique de prévention. Art. L1152-1 du Code du travail ; Art. 222-33-2 du Code pénal.

2 Le professeur David Yamada a été un ardent défenseur de la reconnaissance juridique du harcèlement psychologique en milieu de travail et a rédigé une loi type, le Healthy Workplace Bill, qui a servi de base aux projets de loi présentés dans plusieurs Etats. Voir D. C. Yamada, Crafting a Legislative Response to Workplace Bullying, Employee Rights and Employment Policy Journal, 2000, n 8, p. 475. 


\section{I - LA PROTECTION CONTRE LE HARCÈLEMENT PSYCHOLOGIQUE PAR LES LOIS FÉDÉRALES SUR LES DISCRIMINATIONS}

La source de protection la plus probable des salariés contre le harcèlement psychologique au travail réside dans l'interdiction au niveau fédéral de la discrimination au travail fondée sur des caractéristiques limitativement énumérés. Le titre VII du Civil Rights Act de $1964^{3}$ interdit les discriminations fondée sur la race, le sexe, la religion et l'origine nationale ; la Cour suprême des Etats-Unis a décidé que le harcèlement pour l'un de ces motifs peut constituer une discrimination dans les conditions de travail si certains de ces critères sont remplis ${ }^{4}$. La loi Age Discrimination in Employment Act de $1967^{5}$ et le titre I de l'Americans with Disabilities Act de $1990^{6}$ contiennent des interdictions de discrimination similaires, et donc de harcèlement, fondées sur l'âge - à partir de 40 ans et sur le handicap. Le harcèlement ou l'intimidation qui ne repose pas sur l'un des motifs figurant dans la loi anti-discrimination n'est généralement pas illégal, du moins en vertu $d^{\prime} u n e$ loi fédérale ${ }^{7}$. Autrement dit, les salariés victimes de harcèlement psychologique en raison de leur race, de leur sexe ou d'un autre motif visé par la loi sur les discriminations disposent d'une base légale pour contester le harcèlement dont ils sont victimes, tandis que les salariés harcelés pour une autre raison ne peuvent pas bénéficier de la protection juridique apportée par les lois contre la discrimination.

Même les salariés qui peuvent démontrer qu'ils ont été victimes de harcèlement en raison des motifs visés par les lois antidiscriminatoires doivent toujours satisfaire à d'autres exigences pour que ce harcèlement soit considéré comme une violation de la loi fédérale. En particulier, les salariés doivent être en mesure de démontrer qu'ils perçoivent subjectivement le harcèlement ou l'intimidation comme offensant ou abusif, et que la conduite est objectivement hostile ou systématique de sorte qu'une personne raisonnable dans leur situation trouverait la conduite hostile ou abusive, et que les termes et conditions de leur emploi ont été modifiés de manière discriminatoire ${ }^{8}$. En conséquence, même l'intimidation motivée par des critères protégés par les lois antidiscriminatoires ne sera illégale que si ce comportement est très grave ou s'il se poursuit sur une certaine durée.

342 U.S.C. secs. 2000 e to 2000 e- 17.

4 Meritor Savings Bank v. Vinson, 477 U.S. 57 (1986)(«Sans aucun doute, quand un superviseur harcèle sexuellement un subalterne en raison de son sexe, ce superviseur "exerce une discrimination" en raison du sexe $»)$.

529 U.S.C. secs. 631 to 633 a.

$6 \quad 42$ U.S.C. secs. 12101 à 12103, 12111 à 12117.

7 Voir Johnson v. City University of New York, 48 F. Supp. 3d 572, 574 (S.D.N.Y. 2014) («L'intimidation et le harcèlement n'ont pas leur place sur le lieu de travail, à moins qu'ils ne soient motivés par l'appartenance de la victime à une classe protégée, ils ne constituent pas la base d'une action en vertu du titre VII. ») ; Watkins v. First Student, Inc., 2018 WL 1135480, * 15 (S.D.N.Y. 2018) ("Quand [les tribunaux] disent que le titre VII, et les lois locales et nationales correspondantes, ne sont pas un code de civilité, ils disent que même si la méchanceté et l'intimidation rendent un environnement de travail abusif, il n'y a pas violation de la loi à moins que cette méchanceté et ce harcèlement ne soient enracinés dans... la discrimination [fondée sur une caractéristique protégée] »).

8 Voir Harris v. Forklift Systems, Inc., 510 U.S. 17, $21-23$ (1993). Voir également Davis-Garett v. Urban Outfitters, Inc., 2017 Fair. Empl. Prac. Cases (BNA) 342,705, * 4 (S.D.N.Y. 2017) ("Le simple harcèlement n'est pas suffisant pour affirmer qu'un environnement de travail est hostile »). 
Un certain nombre de tribunaux ont décidé que des salariés ont allégué ou prouvé l'existence d'un harcèlement suffisamment grave ou envahissant pour être considéré comme créant un milieu de travail hostile et, par conséquent, illégal en vertu des lois antidiscriminatoires ${ }^{9}$.

La loi Occupational Safety and Health Act (sur la sécurité et la santé au travail) constitue une autre source potentielle de protection au niveau fédéral contre le harcèlement psychologique, car elle comporte une disposition générale sur les devoirs selon laquelle «[chaque employeur (...) doit fournir à chacun de ses salariés un emploi et un lieu de travail qui ne comportent aucun danger reconnu, causant ou pouvant causer la mort ou des dommages physiques sérieux à ses salariés ${ }^{10}$. " Bien que le harcèlement au travail soit reconnu comme une forme de violence au travail qui peut avoir des effets aussi bien physiques que psychologiques ${ }^{11}$, l'Occupational Safety and Health Administration (l'Administration en charge de la sécurité et de la santé) ne semble pas considérer le harcèlement psychologique au travail comme un problème relevant de sa compétence générale. Au lieu de cela, l'Agence estime que "les brimades, l'intimidation et le harcèlement ne satisfont généralement pas [aux] critères » considérés comme une cause avérée ou possible de dommages physiques ${ }^{12}$.

L'Administration en charge de la sécurité et de la santé au travail n'a pas imposé d'obligation aux employeurs quant à la prévention du harcèlement psychologique au travail ${ }^{13}$. Dans ses procédures d'application de la loi sur l'exposition professionnelle à la violence au travail, l'Agence indique que « I'OSHA ne devrait généralement pas initier une inspection en cas de menaces personnelles ou émanant de collègues ", mais devrait plutôt considérer l'opportunité d'une inspection au cas par cas, en fonction de la prévisibilité

9 Voir, par exemple, Equal Employment Opportunity Commission v. National Education Association, Alaska, 422 F.3d 840 ( $9^{\text {th }}$ Cir. 2005) (le harcèlement de trois femmes sur leur lieu de travail, $y$ compris en leur faisant des remarques grossières et des menaces physiques, pourrait motiver une plainte en vertu du titre VII) ; Nesbitt v. University of Maryland Medical Center, 2013 WL 6490275 (D. Md. 2013) (un comportement de harcèlement et d'humiliation pendant deux ans sous forme d'attaques hebdomadaires, y compris en criant sur la plaignante devant les autres salariés et en la traitant de manière méprisante, a été suffisant pour déclarer le harcèlement fondé sur le sexe et l'âge de la victime).

1029 U.S.C. sec. 654(a)(1).

11 L'Administration en charge de la sécurité et de la santé au travail définit la « violence au travail » comme « tout acte ou menace de violence physique, de harcèlement, d'intimidation ou tout autre comportement perturbateur menaçant sur le lieu de travail, y compris les menaces et la violence verbale. " United States Department of Labor, Occupational Safety and Health Administration, Safety and Health Topics, https://www.osha.gov/SLTC/workplaceviolence

Voir également Garity v.APWU-AFL-CIO, 2017 L.R.R.M. (BNA) 286,035, * 3 (D. Nev. 2017) (excluant le témoignage d'un expert en harcèlement au travail, qui aurait témoigné que l'intimidation au travail peut causer une détresse mentale et physique, car le témoignage ne serait pas utile au jury "parce qu'il ne concerne pas des choses dépassant les connaissances communes du profane»).

12 United States Department of Labor, Occupational Safety and Health Administration, Directive $n^{\circ}$ CPL 02-01-058, Enforcement Procedures and Scheduling for Occupational Exposure to

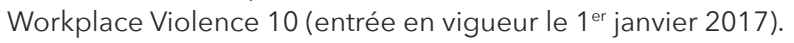

13 Voir Memorandum for Regional Administrators de Richard E. Fairfax, Directeur, Directorate of Enforcement Programs (June 4, 2004) (faisant référence aux lignes directrices fournies aux employeurs sur la violence au travail et notant que l'omission de mettre en œuvre les directives «n'est pas une violation, ou une preuve d'un manquement à l'obligation de veiller à la santé du salarié »). 
et de la gravité des cas, et du fait que l'employeur a pris ou non des mesures pour faire face à cette menace. Ces procédures indiquent que si les directeurs régionaux prennent connaissance de « cas pouvant être qualifiés de harcèlement ou d'intimidation, ils devraient envisager de renvoyer le plaignant devant l'autorité gouvernementale compétente ", soit l'Equal Employment Opportunity Commission (Commission pour l'égalité des chances) soit le National Labor Relations Board (Conseil national des relations du travail) ${ }^{14}$. Ces procédures montrent que l'Administration en charge de la sécurité et de la santé au travail considère généralement que le harcèlement au travail n'est pas un problème allant à l'encontre de la Loi sur la sécurité et la santé au travail, mais un problème pouvant relever d'autres régimes juridiques. D'autre part, les lignes directrices semblent reconnaître que les incidents de harcèlement psychologique au travail suffisamment graves et prévisibles de la part de l'employeur pourraient constituer un manquement à ses devoirs, surtout si l'employeur n'a pas pris de mesures pour y remédier.

\section{II - LES LOIS DES ETATS EN MATIÈRE DE HARCÈLEMENT PSYCHOLOGIQUE AU TRAVAIL}

Bien que la loi fédérale ne traite pas spécifiquement du harcèlement psychologique au travail, il existe dans certains Etats des lois propres à cette question. La Californie, le Tennessee et l'Utah, en particulier, ont promulgué des lois pour lutter contre le harcèlement psychologique au travail ${ }^{15}$, mais aucune de ces lois n'interdit expressément le harcèlement psychologique au travail ou n'offre une base pour une action en justice de salariés victimes de harcèlement au travail.

La loi californienne prévoit que dans cet Etat, les employeurs déjà tenus de dispenser une formation sur le harcèlement sexuel aux salariés chargés de la supervision sont également obligés d'inclure, dans le cadre de cette formation, la prévention des comportements abusifs. La loi définit un « comportement abusif » comme le " comportement au travail d'un employeur ou d'un salarié faisant preuve de malveillance qu'une personne raisonnable trouverait hostile, injurieux et sans rapport avec les intérêts professionnels légitimes d'un employeur ${ }^{16}$. La loi prévoit qu'une omission relative à cette obligation de formation ne suffit pas « en soi » pour rendre l'employeur responsable en cas de plainte pour harcèlement sexuel d'un salarié, d'un ancien salarié ou d'un candidat à un poste, de la même manière que le respect de la loi par l'employeur ne le dégage pas de toute responsabilité en cas

14 United States Department of Labor, Occupational Safety and Health Administration, Directive $n^{\circ}$ CPL 02-01-058, Enforcement Procedures and Scheduling for Occupational Exposure to Workplace Violence 7 (entrée en vigueur le 1er janvier 2017).

15 Un autre Etat, Puerto Rico, a adopté en 2014 une loi déclarant le 9 octobre « Journée de l'unité » afin de sensibiliser le public à l'intimidation dans « les écoles, les lieux de travail et les communautés ». 2014 P.R. Laws $n^{\circ} 7$ (approuvé le 25 juin 2014) ; I L.P.R.A. sec. 5271.

16 La disposition californienne relative au « comportement abusif » a été promulguée en 2014. 2014 Cal. Legis. Serv. ch. 306 (déposée le 9 septembre 2014). Cal. Gov't Code sec. 12950.1(b), (h)(2). La loi définit en outre la conduite abusive de la façon suivante : " Une conduite abusive peut inclure des injures répétées, telles que des remarques désobligeantes, des insultes et des épithètes, une conduite verbale ou physique qu'une personne raisonnable trouverait menaçante, intimidante, ou humiliante, le sabotage gratuit ou la mise à mal de l'exécution du travail d'une personne. Un seul acte ne constitue pas un comportement abusif, à moins d'être particulièrement grave et flagrant ». 
de plainte pour harcèlement sexue ${ }^{17}$. Ainsi, bien que la loi californienne œuvre pour que les employeurs et l'encadrement des entreprises soient plus conscients de la nécessité de prévenir le harcèlement psychologique dans le travail, elle n'impose pas pour autant à l'employeur une responsabilité en cas d'harcèlement psychologique.

La loi de I'Utah traitant du harcèlement psychologique au travail met aussi l'accent sur la formation plutôt que sur l'interdiction de cette conduite. Cette loi a modifié la législation sur la gestion du personnel de l'Etat de I'Utah afin d'obliger le Département de la gestion des ressources humaines en Utah à dispenser une formation aux managers et aux salariés des agences sur la prévention des comportements abusifs sur le lieu de travail. Cette loi autorise également ce service à élaborer des politiques contre la conduite abusive dans le travail. La loi définit un " comportement abusif » comme «le comportement verbal, non verbal ou physique d'un salarié envers un autre salarié qui, selon sa gravité, sa nature et sa fréquence, serait considéré par une personne raisonnable... comme destiné à provoquer un harcèlement psychologique, une humiliation ou une détresse injustifiée [ou]... à l'origine de dommages physiques ou psychologiques graves ${ }^{18}$ ". La loi de l'Utah dispose qu'elle ne crée pas un droit d'action en justice, pas plus qu'elle n'élargit ou ne diminue les droits auparavant disponibles, ou qu'elle n'élargit ou ne diminue les motifs de sanction, mais qu'elle « n'exempte pas ou ne dégage pas non plus une personne d'une responsabilité, d'une obligation ou d'une sanction éventuelle, en vertu d'une autre loi de l'Etat ${ }^{19}$ ". A l'instar de la loi californienne, la loi de l'Utah n'impose pas à l'employeur une responsabilité en cas de harcèlement psychologique au travail ou n'influe en rien sur la responsabilité de l'employeur vis-à-vis de ce comportement.

La loi du Tennessee sur le harcèlement psychologique dans le travail, connue sous le nom de Healthy Workplace Act (loi pour un environnement de travail sain), prévoit que la Tennessee Advisory Commission on Intergovernmental Relations (la Commission consultative du Tennessee sur les relations intergouvernementales) établisse une politique type pour les employeurs visant à prévenir les comportements abusifs sur le lieu de travail. Cette loi dispose que l'employeur qui adopte cette politique type doit « ne faire l'objet d'aucune poursuite pour comportement abusif d'un salarié ayant entraîné une souffrance mentale par négligence ou intentionnellement », étant entendu qu'une telle politique ne limite pas la responsabilité personnelle d'un salarié en cas de comportement abusif ${ }^{20}$. La loi définit un "comportement abusif » comme l'ensemble des " actions ou omissions qu'une personne raisonnable, selon la gravité, la nature et la fréquence de ce comportement, estime qu'un salarié a été soumis à un milieu de travail abusif, se traduisant par : la violence verbale répétée sur le lieu de travail, y compris les remarques désobligeantes, les insultes

17 Cal. Gov't Code sec. 12950.1(e).

18 Cette loi a été promulguée en 2015. 2015 Utah Laws ch. 211 (entrée en vigueur le 1er juillet 2015). Utah Code Ann. sec. 67-19-44. La loi prévoit également que «[un] acte unique ne constitue pas un comportement abusif, à moins qu'il ne soit particulièrement grave et flagrant ». Le Code administratif de I'Utah dispose que " [L]a politique de l'Etat de I'Utah consiste à fournir un environnement de travail dépourvu de tout comportement abusif » et contient des informations sur les procédures de plainte et d'enquête en cas de conduite abusive sur le lieu de travail. U.A.C. R477-16.

19 Utah Code Ann. sec. 67-19-44(7).

20 La loi du Tennessee a été adoptée en 2014. 2014 Tenn. Laws ch. 997 (approuvé le 22 mai 2014). Tenn. Stat. sec. 50-1-501 to 50-1-505. 
et les épithètes, une conduite verbale, non verbale ou physique de nature menaçante, intimidante ou humiliante sur le lieu de travail, ou le sabotage ou la détérioration du travail d'un salarié sur le lieu de travail ${ }^{21} »$.

La loi du Tennessee ne s'applique qu'aux agences gouvernementales locales et étatiques. Les employeurs du secteur privé ne sont pas visés par la lois. Bien que le personnel de la Commission ait élaboré une politique type, les instances dirigeantes de cet organisme refusèrent de l'adopter. Toutefois, le Département des Ressources humaines du Tennessee a adopté une politique sur la conduite abusive en milieu de travail. Cette politique préconise que « la politique de l'Etat du Tennessee soit de fournir un environnement de travail exempt de tout comportement abusif » et que cet Etat « ne tolère sur le lieu de travail aucun comportement abusif ou qui perturbe le milieu de travail ». Toutefois, cette politique précise également qu'elle " n’a pas pour objet de définir les conditions contractuelles de travail entre l'Etat et ses salariés, les demandeurs d'emploi ou les parties en relation d'affaire avec l'Etat ", que " cette déclaration constitue uniquement la politique de l'Etat de Tennessee » et que la violation de cette politique «ne signifie pas que la conduite viole les lois fédérales et/ou d'Etat ${ }^{22} »$. En conséquence, la loi du Tennessee n'impose aucune obligation légale aux employeurs publics en matière de harcèlement psychologique et peut en fait, dans certaines circonstances, les dégager de toute responsabilité dans les affaires de harcèlement psychologique au travail.

Certains salariés ont demandé aux tribunaux de reconnaître la validité d'une allégation de harcèlement psychologique au travail en Common law en l'absence d'une loi. Ces demandes ont échoué, les tribunaux ne voulant pas reconnaître la validité d'une telle allégation en l'absence de mesures législatives. Par conséquent, les juges ont généralement refusé de reconnaître les demandes en responsabilité délictuelle fondées sur le harcèlement psychologique au de travail ou une action pour licenciement injustifié fondée sur une politique publique contre le harcèlement psychologique au travail ${ }^{23}$.

II existe d'autres bases légales, issues de la Common law de plusieurs Etats, qui ont été invoquées afin d'imputer aux employeurs une responsabilité dans les affaires de harcèlement psychologique au travail. Bien que certains salariés aient fait valoir avec succès des allégations concernant des voies de fait et des violences physiques réelles ou des menaces ${ }^{24}$, la demande la plus susceptible d'être invoquée pour les préjudices psychologiques de harcèlement au travail est une allégation de souffrance émotionnelle

21 Tenn. Stat. sec. 50-1-502(1).

22 Tennessee Department of Human Resources Policy n 17-001 (entrée en vigueur le 15 mars 2017).

23 Voir, par exemple, Connearney v. Main Line Hospitals, Inc., 2015 WL 9302912, * 6 (E.D. Pa. 2015) (le tribunal a refusé de « recevoir une action en Common law en Pennsylvanie pour harcèlement») ; Ramseur v. Perez, 962 F. Supp. 2d 21, 30 (D.D.C. 2013) (rejetant la demande en responsabilité délictuelle du plaignant pour « intimidation dans le travail » au motif qu'il n'y a pas de «fondement de droit commun indépendant pouvant être reconnu comme une « intimidation dans le travail »); Jaber v. FirstMerit Corp., 2017 -Ohio- 277, 81 N.E.3d 879, 891-892 (Ohio App. 2017) (le tribunal a refusé d'élargir le champ d'application de la loi pour reconnaître une plainte pour violation de la politique publique sur la base du harcèlement).

24 Voir Raess v. Doescher, 883 N.E. 2d 790, 793-794 (Ind. 2008) (un membre du personnel hospitalier a déclaré avoir été victime d'une agression de la part d'un médecin en raison de l'action agressive et rapide du médecin qui s'est avancé vers le plaignant, les poings serrés, le regard menaçant, le visage congestionné, les veines saillantes, en hurlant et en jurant, puis en déclarant « vous êtes fini, vous êtes viré »). 
infligée intentionnellement ${ }^{25}$. Afin de justifier sa plainte pour avoir subi une souffrance émotionnelle intentionnelle, le salarié doit établir l'existence d'une conduite extrême et outrageante susceptible de provoquer une détresse émotionnelle grave. Le degré à atteindre pour qu'un comportement soit jugé extrême et outrageant et justifie une allégation selon laquelle la détresse émotionnelle a été infligée intentionnellement a été décrit comme étant «si atroce qu'il dépasse les limites de la décence et qu'il est totalement intolérable pour la communauté civilisée » et conduirait " un membre ordinaire de cette communauté à s'exclamer "c'est scandaleux » ${ }^{26}$ ! Par conséquent, seules les formes les plus extrêmes de harcèlement psychologique sont susceptibles de faire l'objet d'une action dans le cadre d'une plainte en responsabilité civile.

Les tribunaux qui ont examiné des demandes en réparation sur le fondement de la souffrance émotionnelle infligée intentionnellement ont exprimé leur désaccord sur le fait de savoir si de telles allégations peuvent être généralement reconnues dans le contexte du travail. Certains tribunaux ont laissé entendre que le harcèlement psychologique dans le travail est un phénomène assez répandu si bien qu'il ne serait pas nécessaire que le comportement soit " extrême " pour prouver que la détresse émotionnelle est infligée intentionnellement. Certains tribunaux ont même laissé entendre que « dans le travail, les gens devraient raisonnablement s'attendre à éprouver un certain niveau de détresse émotionnelle, voire une détresse émotionnelle importante, en raison de certains comportements ${ }^{27}$ ". D'un autre côté, certains tribunaux ont suggéré que l'existence d'un déséquilibre inhérent à la relation de travail peut accroître la probabilité d'une allégation de souffrance émotionnelle intentionnelle fondée sur « un schéma de harcèlement délibéré et répété sur une certaine durée ${ }^{28} »$.

Il n'y a pas de cohérence entre les jugements rendus par les tribunaux sur la question de savoir si le harcèlement au travail doit être suffisamment grave pour justifier une demande en réparation pour souffrance émotionnelle intentionnelle. Par exemple, certains tribunaux ont jugé que «le harcèlement psychologique et l'ostracisme » par des collègues, consistant à " avoir un comportement de nature à isoler quelqu'un, des commentaires indélicats et des conversations hostiles et désagréables ", ne suffisent pas à remplir les critères définissant un comportement extrême et outrageant ${ }^{29}$. Mais certains tribunaux ont permis que les plaintes des salariés pour détresse émotionnelle infligée intentionnellement

25 Voir Raess v. Doescher, 883 N.E.2d 790, 799 (Ind. 2008) (En discutant de l'instruction proposée sur «l'intimidation en milieu de travail», le tribunal a noté que « l'intimidation en milieu de travail pouvait être considérée comme une forme d'infliction intentionnelle de détresse émotionnelle ».

26 Kearney v. Orthopaedic and Fracture Clinic, P.A., 2015 WL 5194832, * 6 (Minn. App. 2015). Voir également Restatement (Second) of Torts sec. 46 cmt. d (1965); Restatement (Third) of Torts sec. $46 \mathrm{cmt} . d$ (2012).

27 Espinoza v. The Computer Co., 2015 WL 1014865 (Conn. Super. Ct. 2015). Voir également Woodward v. Elizabethtown Community and Technical College, 2015 WL 4464100, * 6 (W.D. Ky. 2015) ( "Le lieu de travail est trop souvent le cadre d'insultes et d'indignités, mais la loi ne remédie pas à toutes les actions grossières et décourageantes commises par les employeurs »).

28 Northern v. State of Louisiana, 2015 WL 6839998, * 6 (La. App. 2015).

29 Kearney v. Orthopaedic and Fracture Clinic, P.A., 2015 WL 5194832, * 7 (Minn. App. 2015). Voir également Britton v. Athenahealth, Inc., 31 Mass. L. Rptr. 182, 2013 WL 2181654, * 5 (Mass. Super. Ct. 2013) (l'intimidation, y compris l'envoi de courriels hostiles et le sabotage des performances au travail du salarié, ne répondait pas aux critères définissant un comportement extrême et outrageant). 
soient entendues et soumises à la décision d'un jury, autorisant ainsi les jurés à conclure que le harcèlement psychologique était suffisamment extrême et scandaleux pour être illégal en vertu de cette action en responsabilité délictuelle ${ }^{30}$.

\section{Conclusion}

Aux Etats-Unis, la loi n'a pas suivi le même rythme que dans d'autres pays en ce qui concerne la reconnaissance des demandes fondées sur le harcèlement psychologique au travail. II semble que cet échec soit imputable à deux préoccupations différentes exprimées par les législateurs et les tribunaux. La première est que la reconnaissance d'une allégation de harcèlement au travail imposerait des " codes de civilité " sur les lieux de travail et limiterait ainsi l'action des employeurs et des managers dans leurs relations avec les salariés ${ }^{31}$. La seconde est que le fait de tenir les employeurs responsables de préjudices psychologiques les obligerait à payer le coût de ce qui peut être considéré comme un préjudice invérifiable. Peut-être la reconnaissance du coût personnel et organisationnel du harcèlement psychologique en milieu de travail pourrait-elle conduire les employeurs, les tribunaux et les législateurs à ressentir le besoin de s'attaquer à ce problème envahissant dans le milieu du travail.

30 Voir, par exemple Willis v. Career Education Corp., 31 A.D. Cases (BNA) 1293, 2015 WL 3859191, * 13 (N.D. III. 2015) (I'intimidation au travail pourrait franchir le seuil d'une conduite extrême et outrageant); Espinoza v. The Computer Co., 2015 WL 1014865, * 1-2 (Conn. Super. Ct. 2015) (le tribunal a jugé que des esprits raisonnables pouvaient ne pas être d'accord sur le fait que l'intimidation à laquelle le salarié était soumis par son superviseur ait été extrême et outrageant).

31 Voir Oncale v. Sundowner Offshore Services, Inc., 523 U.S. 75, 81 (1998) (s'intéressant à l'idée de transformer le Titre VII en un « code de civilité générale »). 